\title{
AFIRMACIÓN TEÓRICA EN LA CONTEMPORANEIDAD DE LA ENSEÑANZA ARTÍSTICA LATINOAMERICANA
}

\author{
Dr. Aurelio A. Horta Mes $\Lambda$ \\ Doctor en Ciencias sobre Arte \\ Vicerrector Docente del Instituto Superior de Arte. \\ Profesor: Cultura Cubana, Literatura \\ Universal y Cubana, Política Cultural
}

En el número cuatro de los remedios que se describen en las Relaciones que hicieron algunos religiosos sobre los excesos que había en lndias y varios memoriales de personas particulares que informan de cosas que convendrían remediar, de Fray Bartolomé de las Casas, que sabemos se convirtieron además en orientaciones para el desempeño de un supuesto buen gobierno en nuestras tierras, escribe el controvertido dominico:

Que hecha la tal suspensión, en el primer remedio dicha, se trata de la solicitud de que no sean los indios prontamente muertos por causa de sus servicios y trabajos según la costumbre de los españoles, vuestra reverendísima señoria mande a pregonary hacer entender, con lenguas de la tierra a todos los indios de las Islas, cómo les quiere hacer nueva merced, en que no han de servir como solían, ni ser sujetos a los cristianos de la manera pasada, sino de otra que mejor pueden sufrir y cómo no mueran y vivan. Y esto aprovechará para consolación de ellos y para esperar que habrá su mal pasado remedio, porque cierto, sin pensar que nunca hasta que mueran han de salir de aquel pésimo tratamiento, como han visto sus pasados, según ellos son débiles de corazón, basta que desesperen, como muchos se han muerto como con cierto rejalgar que tienen y gran cantidad de ellos, o se mueran de pensamiento, como también ha acaecido. ${ }^{29}$

Todavía mucho nos cuesta eso de "no ser sujetos a los cristianos de la manera pasada". y Las Casas, por razones muy 1ógicas clase y encargo, no supuso que más tarde, la misma empresa conquistadora colonizadora y la importación de negros. representarían en estas tierras, un mundocrisol que ya bien hemos aprendido desde que el sabio Fernando Ortiz. nos propusiera como suma categoría, la de transculturación. Por lo que ya tampoco nos concierne aquello de "débiles del corazón a causa de la desesperanza", o la posibilidad de "morir de pensamiento", ya que la misma huella del fraile y apóstol de los indios, nos libró -si es que las hubo-, de esas variables del carácter, debido a que ha sido la voluntad de sentimientos comunes, y una probada faena de pensar la que ha hecho posible desde hombres como Hatuey, nuestra convocatoria a la vida.

29 Las Casas, Bartolomé de, en: "Relaciones que hicieron algunos religiosos sobre los escesos que había en Indias, y varios memoriales de personas particulares que informan de cosas que convendrán remediar", tomado de: Almodovar Muñoz, Carmén. Antología crítica de la historiografía cubana (época colonial). Ciudad de la Habana, Editorial Pueblo y Educación 1986, p,54. 
Esa misma fe de Las Casas, y su rara perspectiva, que lo ha hecho distinguir más allá de nuestra historia nos refiere, desde un primer momento, a su defensa por lo que hoy consideramos nuestra identidad cultural, y que constituye esa noción por el respeto a la convivencia entre diferentes tipos de creencias y sociedades, y otra muy precursora visión, que es aquella que reclama la necesidad de una conservación del patrimonio arqueológico y artístico de una cultura para los otros hombres desde el siglo XV hasta el XX, insospechada pero decididamente necesaria también para ellos.

Es válido reconocer, entonces, esa inmerecida desobediencia de S.A. a los "remedios" de aquel utopis-

ta, que nos dispuso la problematización no solo del natural derecho a la existencia, que en realidad es el de gozar una libertad individual, sino el de proponernos un primer modelo para el crecimiento. $\mathrm{Y}$ próspero de una sociedad, que justo en su milenaria confron- tación, se precisa por una autónoma fundamentación de su pensamiento y acción.

Ante todo, si concebimos esta tarea como algo distante del mismo acarreo social, no es posible llegar a conclusión alguna. Los derroteros de la cultura no son más que la explicitación del engendro de la historia de la sociedad, por tanto, el motivo que nos reune nos debe, en primer lugar, hacer reflexionar acerca de cuales deben ser prioritariamente las leyes de formación de una personalidad como portadora de una cultura.
En este caso, la enseñanza artística latinoamericana se debe a una realidad única, por cuanto su orientación no puede ser otra que la de alcanzar como objetivo primordial, el de un desarrollo sociocultural de sus pueblos. De ahi la conciencia de su unicidad y particularidad metodológica. No se trata de continuar esbozando proyectos en relación con la eficacia artística, se tratz. de sentar las bases de una fundamentación científica, que nos permita más que exhibir, probar cuales deben ser los diseños, y los métodos que avalen las especificidades del aprendizaje de las artes, en un mundo cuya experiencia vital, y cuyo sentido de predisposición creadora ha sido por naturaleza de vocación humanista.

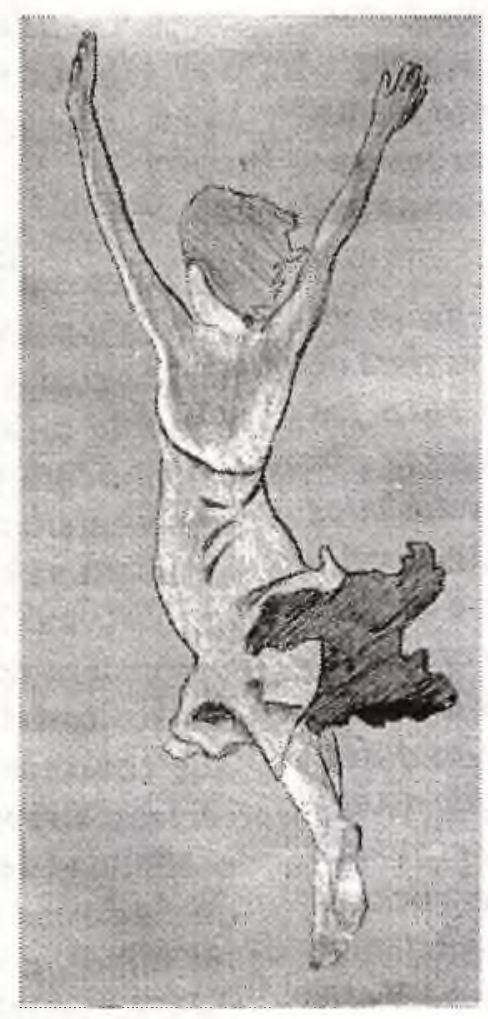

No es posible una acción fragmentada de la enseñanza artística en América Latina. Dígase más, no es confiable un enfoque partícular, regional de la pedagogía especializada en tanto especialización, ya que una condición primera de cualesquiera que fueran nuestras intenciones académicas, deben regirse por principios de una ética artística que por supuesto presupone una ética ciudadana que solo el dominio de una cultura artística determinada puede enrumbar y diseñar satisfactoriamente.

Ya la premonición de José Martí, nos había enseñado que: "ni el libro europeo, ni el libro yanqui, daban la clave del enigma hispanoamericano" ${ }^{30}$. La anchura y vuelo

30 Martí, José. "Nuestra América", en: Obras completas. La Habana. Editorial de Ciencias Sociales. 1975, t. 6, p. 20. 
seguro de la escuela de arte no puede cumplir con su verdadero papel de desarrollar el talento y emancipar la voluntad creadora, si se ciñe a moldes generales que no le competen, es decir, que no la especifican. El reclamo de una Justipreciación de la enseñanza del arte en América Latina, tal y como sentimos, debe alcanzar a reconocer nuestras sociedades, depende en gran medida, del espacio que ésta ocupe no sólo en escenarios y concursos, sino en la demostración científica de su determinante papel en la conservación y valuación de nuestro patrimonio, y en la influencia que ejerza en el trabajo de modelación social, a partir de sus instituciones y perfiles artísticos, cuestión ésta que demanda de un inminente estudio del pensamiento culturológico latinoamericano, de su descripción e interpretación a la luz de nuestros intereses artísticos-pedagógicos.

Son estas, las más generales consideraciones para arribar nuestro caso, desde la dominante de nuestras acciones en la pedagogía artística al estudio del pensamiento y teoría artística carpenterianos.

Alejo Carpentier ocupa en el ámbito de la cultura latinoamericana, un espacio de alta consideración. Así convenido la dimensión de su praxis artístico-literaria ha sido y es motivo de los más variados estudios, concentrados fundamentalmente en la reinterpretación de su fundacional obra narrativa, como testimonio de la generalizada aceptación de su maestria profesional. Esta circunstancia ha propiciado con justeza en su prioridad, un vasto estudio de incuestionable interés, referido fundamentalmente a la importancia de su novelística en el contexto latinoamericano.

Urge así la necesidad de revelar la contribución de la exégesis carpenteriana al discernimiento de las variables específicas de la práctica artística, explícitas de manera constantes en su profusa crítica, conferencias, ensayos y otros tipos de actividades de fundamentación teórica.

Un primer acercamiento a este propósito nos remite, por supuesto, a esclarecer cómo al asumir el ejercicio artístico lo hacemos a partir de su condicionamiento sociocultural, y que lo diferencia por tanto de otras muchas prácticas humanas. Por tanto, al referimos en adelante a la "práctica artística" consideramos la misma en su dependencia a aquellos caracteres que denotan su virtual comportamiento y según las premisas de si la misma esta determinada o no por: a) la actuación profesional consciente, b) el mérito de su calidad, c) la definición de su intención estática. (Véase-140-Williams. Raymond-Cultura. Sociología de la Comunicación y del Arte.

Este criterio basado en el principio de que la práctica del arte es, también, un proceso histórico y social al igual que otras prácticas humanas, inclusivas además del factor estético, es del todo muy propio en relación con el presente objeto de estudio. si convenimos que en la especificidad de la actuación social en Carpentier, estas diferentes actividades constituyen funciones proteccionistas de su voluntad profesional de escritor.

Otro aspecto complementario a considerar en este asunto, es el mismo hecho de ser el crítico Carpentier, un portavoz exclusivo de los procesos de creación, percepción y valoración de la práctica artística en la primera mitad de este siglo, cuestión que fundamenta su estudio acerca del desarrollo y componentes fundamentales de nuestra cultura artística y literaria. 
El juicio crítico carpenteriano sobre la artisticidad, es una condición sine qua non de su multifuncionalidad expresiva, que revela la singular concepción de Carpentier acerca de la naturaleza e interpretación del hecho artístico, al trasvasar este los límites en general establecidos para distinguir los espacios de la crítica y una peculiar concepción de este ejercicio que lo coloca en la tradición de los "raros".

En este sentido vale la consulta al entológico estudio de Cintio Vitier. La crítica literaria estética en el siglo XIX cubano, donde se expone de manera acuciosa las principales ideas que abord6 la crítica cubana, donde se expone de manera acuciosa las principales ideas que abordó la crítica cubana, donde nos define además de su orientación "sociológica y patriótica", el nivel de la misma al servir de base a la modelación y rumbo de nuestra autoctonía cultural, a partir del punto nodal de José Martí que "...logró romper el círculo vicioso de la colonia la alternativa Madrid París y trascender sus antinomias metodológicas y culturales para legarnos un ejemplo de "ejercicio de criterio" radicalmente libre y0 tan vigente hoy como toda su palabra revolucionaria, ejemplo vivo y proteico basado en dos principios fundamentales- la raíz americana y la participación universal".

Resulta la crítica uno de los más incisivos campos que revelan el aporte de nuestra intelectualidad al desarrollo de la cultural al discriminar sobre nuestro específico acontecer cultural entiéndase latinoamericano, propiciando una autoconciencia sobre nuestra identidad artística, escena en la que el profuso esciitior cubano ocupa un lugar de excepción.

En Carpentier nos encontramos frente a una visión sui generis del hecho artístico que nos

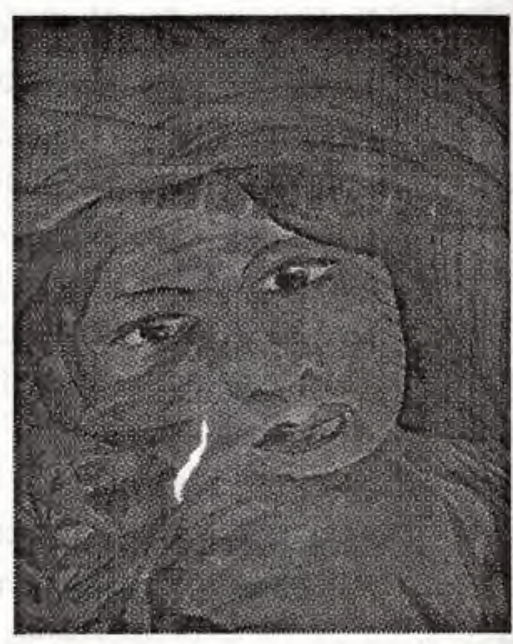

remite al unísono a reconocer en el meollo de su teoría la polifuncionalidad de su método de creación hasta el momento estudiado de forma preferente en su obra narrativa.

Nos referimos en este caso a su política de lo real maravilloso americano donde es posible hallar en su dialéctica una constante referencia al proceso de creación artística, evidencia motodológica de la intertextualidad. Justamente, en la voluntad del escritor Carpentier por describir el objeto y fundamentación de su novela, arriba el teórico a juicios particulares de una máxima generalización acerca de la actividad de creación en diferentes dominios artísticos.

La atención carpenteriana se concentra en un diálogo crítico entre la herencia de la tradición occidental, de la cual forma parte América y sus "artistas funcionales" para la realidad latinoamericana. Un tema, que por demás centraliza el problema de la cultura americana y su condición dialógica, algo que ya se halla en el interés de la intelectualidad latinoamericana desde el siglo XIX, como es en el caso de José Agustín Caballero.

En el pensamiento carpenteriano se advierte un núcleo fundamental de razonamientos referidos a la creación artística, que se corresponden con la 
dialéctica y especialización de su discurso, lo cual ha favorecido la idea acerca de la presencia en su obra de una "unicidad y constantes carpenterianas" ${ }^{31}$.

Esta posición asumida por el investigador Alexis Márquez puede resultar harto extrema y por ello discutible, ya que en principio, los diez puntos que a manera de invariantes presentan el carácter global y único de la obra objeto de nuestro estudio -según la conclusión de Márquez Rodríguez-, significan un nivel de gradación e intensidad en la novela de Carpentier, al menos en cuanto a los dos aspectos que a nuestro juicio son de máxima consideración en un acercamiento a la praxis artística como es el caso de: A) La inconformidad y perfectibilidad del hombre, y B) La unicidad y globalidad de la cultura universal dado por una integración en la novela, de las ciencias y las artes. Pero no deja de apuntar la tesis del profesor venezolano hacia un dato cierto- la tangible coherencia que, a pesar de apreciables transformaciones, revela determinados aspectos conceptuales en Carpentier, sobre -todo aquellos que determinan la función estético comunicativa de su obra donde es evidente el interés del autor por establecer un sistema de asociaciones culturales que al fin estructuran su discurso y posibilitan a su interlocutor un registro de valores sustanciales de la cultura universal.

La afirmación de una identificación histórico-artística entre creador/obra, se encuentra en Carpentier a partir de su tácita filiación vanguardista, que tiene en cuenta su amplio dominio de las alternativas europeas, y las peculiaridadés no sólo del fenómeno de creación nacional y latinoamericana, sino de las reales; prerrogativas que este proceso demandaba para alcanzar un más amplio reconocimiento universal dentro de las posiciones más progresistas. Intensión de la que es ya deudora su primera novela, revela la problemática que enfrenta el autor de Ecue Yamba O (1933),

Había, pues, que ser nacionalista, tratándose a la vez, de ser "vanguardista". -that's the question... Propósito dificil puesto que todo nacionalismo descansa en el culto a una tradición y el "vanguardismo" significaba por fuerza, una ruptura con la tradición. ${ }^{32}$

El planteamiento en si mismo polémico es a su vez pertinente para un registro en la cultura latinoamericana, donde cabe subrayar como regularidad, la convergencia de los fundamentos sociales de una actuación cívica nacional en vínculo con el pensamiento más relevante de la cultura y el arte occidentales. Este asunto en el caso de Carpentier, constituye definidamente un concepto generador desde la enunciación de lo real maravilloso, al razonar en cuanto a la creación artísticoliteraria en América, cómo ésta no tiene por qué subordinarse a una mimesis del arte tradicional europeo -asunto que ya refiere a una postulación ética-, sino que ésta requiere de una otra volorización de la imagen artística, susceptible de potenciar América no sólo como realidad, sino como referencia cultural.

En mayo de 1927 se hace pública la declaración del Grupo Minorista, y el 12 de septiembre del mismo año. Carpentier inscribe un acento ético-diferenciador a su

${ }^{31}$ Marquéz Rodriguéz, Alexis. Lo barroco y lo real maravilloso en la obra de Alejo Carpentier. Introducción a las obras completas de Alejo Carpentier. México. Editora Siglo XXI. 1982, p, 530.

32 Carpentier, Alejo. Ecue Yamba O. La Habana. Editorial Arte y Literatura, 1977, p11. 
entonces incipiente actividad profesional en el contenido de su carta abierta a Manuel Aznar, que se conoce con la rúbrica "Sobre el meridiano intelectual de nuestra América". En la misma, al indicar los caracteres culturales homogéneos que pueden representar al objeto America en relación con su sujeto creador, Carpentier se refiere a cual debe ser la tarea del joven cronista americano para realmente satisfacer las expectativas de renovación sociocultural a la que aspiran las jovenes generaciones del continente. A partir del postulado de distinguir nuestras realidades americanas y expresarlas según un criterio auténtico. Carpentier se adscribe a los presupuestos martianos que tipifican el género literario de la crónica latinoamericana de muy especial consideración para el presente asunto, en su condición de constituir la misma -según Susana Rotker... "Un sistema de escritura original que supo al fin incorporar tanto lo regional como la cosmopolita mezcla propia de la identidad latinoamericana y de sus procesos de transculturación." 33

Era asunto de sumo interés en la conciencia creadora latinoamericana, "el dilema de una definición espiritual" así como la urgencia de "una apropiación de oficio", siendo aspectos que a partir de la vanguardia entroncan con el ideario éstetico de José Martí en esa raigal revelación de los valores definidores y más altos de nuestra cultura nacional. Carpentier, por supuesto, es sujeto activo de esta común preocupación de la intelectualidad cubana, y su extenso discurso a la sazón es connotativo del desarrollo de la actividad cultural en su conjunto, y muy en particular, de la actividad artística. Una de las más importantes fuentes de investigación en cuestión lo constituyen, sin dudas, el estudio multidisciplinario del periodismo carpenteriano, a partir de una clasificación temática de estos artículos, y de hecho, de un estudio de sus características de dirección comunicativa en cuanto crónicas, sobre todo, atendiendo a la significación que para un estudio de los aspectos conceptuales en la teoría capenteriana representa el período venezolano y la colección Letra y Solfa de El Nacional de Caracas. La importancia y el lugar de esta publicación periódica no sólo en la génesis y desarrollo de la obra de Carpentier, sino en el periodismo latinoamericano. corrobora el criterio de la investigadora Rotker en relación con la crónica como género latinoamericano ya que:

... si como material periodístico las crónicas debían presentar un alto grado de referencialidad y actualidad (la noticia), como material literario han logrado sobrevivir en la historia una vez que los hechos narrados y su cercanía perdieron toda significación inmediata, para revelar el valor textual en toda su autonomía. ${ }^{34}$

Por supuesto, que la afirmación literaria de la crónica así como la singularidad expresiva de la misma en nuestro continente, y justamente es el objeto de estudio de la investigadora venezolana, tiene en José Martí el ejemplo "fundacional de una escritura" dado principalmente porque:

La condición de texto autónomo dentro de la esfera estético-

${ }^{33}$ Rotker, Susana: Fundación de una escritura: las crónicas de José Mártí Ciudad de la Habana. Casa de las Américas, 1992, p. 11.

34 Ibidem, p, 23. 
literanria no depende ni del tema ni de la referencialidad, ni de la actualidad. Ya se ha dicho que muchas de la crónicas modernistas. al desprenderse de ambos elementos temporales, han seguido teniendo valor como objetos textuales en si mismos. Es decir que, perdida con los años la significación principal que las crónicas pudieran tener para el público lector de aquel entonces, son discursos literarios por excelencia. ${ }^{35}$

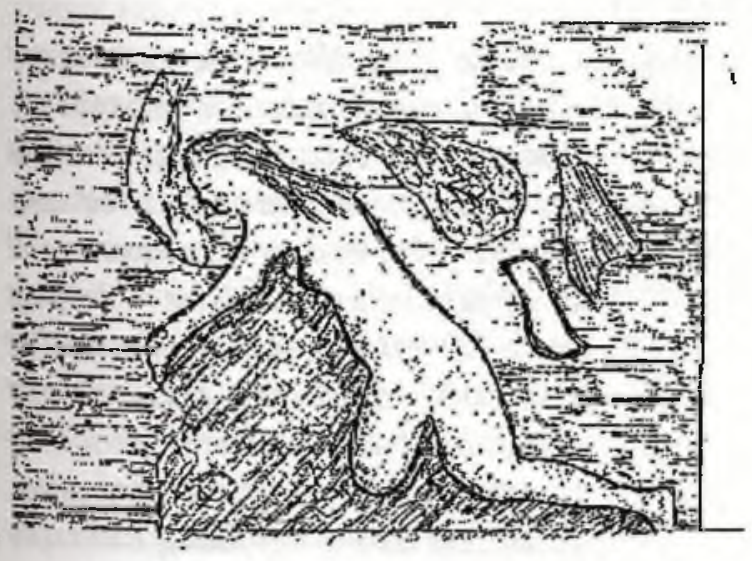

Con profundidad, Roberto Fernández Retamar ha insistido en la pluralidad de funciones, y en la justa ubicación de la personalidad martiana, no sólo como guía de nuestro más radical proceso social, sino por la "genuidad de su literatura" que

... Se encuentran entre las razones fundamentales que han obligado a un replanteo sobre los límites, géneros predominantes y caracteres generales de la literatura entre nosotros. Replanteo para el cual es inútil, vístase del ropaje que se vista, la "falsa erudicción"- pero para el cual, en cambio, es imprescindible la verdadera sabiduría: el conocimiento de lo verdadero en Martí como en nuestra América toda. ${ }^{36}$

Por otra parte, es bien notorio el alcance de la significación de la raíz martiana en las bases ideoéstéticas de la generación minorista donde Carpentier fragua su capacidad ético-artística.

Esa percepción resume un principio presente en toda la obra carpenteriana, basado en la importancia que para la perspectiva de creación significan el talento y el oficio artístico, demostrativo además en esta declaración del teórico de lo real maravilloso.

Ante la desconcertante pobreza imaginativa de un Tanguy, por ejemplo, que desde hace veinticinco años pinta las mismas larvas pétreas bajo el mismo cielo gris, me dan ganas de repetir una frase que enorgullecía a los surrealistas de la primera jornada: Vous qui ne voyes pas pensez a ceux qui voient., ${ }^{37}$

Lo cierto es que Carpentier se orienta hacia una concepción donde el arte y la cultura aparecen en fusión cabal con su entorno en toda su diversidad y dimensión. conformando en esencia el rumbo hacia nuestra identidad cultural $y$ al principio de nuestra predisposición creadora. Así postula:

Historia distinta, desde un principio, puesto que este suelo americano fue teatro

35 Ibidem, p, 136.

${ }^{36}$ Fernandez Retamar, Roberto. "Naturalidad y modernidad en la literatura martiana", en: Letras. Cultura en Cuba. Prefacio y compilación C. Dra. Ana Ballester. La Habana. Editorial Pueblo y Educación. 1989, p.p, 415-442.

${ }^{37}$ Carpentier, Alejo: Ensayos. Ciudad de la Habana. Editorial Letras Cubanas. 1984, p. 77. 
del más sensacional encuentro étnico que registran los anales de nuestro planeta: encuentro del indio, del negro y del europeo de tez más o menos claro destinados, en lo adelante, a mezclarse, entremezclarse, establecer simbiosis de cultura de creencias, de artes populares, en el más tremendo mestizaje que haya podido contemplarse nunca-.. "Tenemos que ser originales" solía decir Simón Rodríguez, maestro del Libertador.. Pero, cuando tales palabras pronunciaba, no había que hacer ya el menor esfuerzo por ser originales pues éramos, ya, originales, de hecho y de derecho, mucho antes de que el concepto originalidad se nos hubiese ofrecido como meta. ${ }^{38}$

Los cauces de estas consideraciones explicitan una conciencia artística reconocedora de su actividad teórico-práctica al asumir no sólo el conocimiento real de las potencialidades de capacidad creadora del artista latinoamericano, sino al insertar ese proceso en un sistema valorativo de códigos universales, al que responde en definitiva la savia martiana, transcendida en la intelectualidad cubana a la que pertenece Carpentier. Juan Marinello, por ejemplo, refiere:

... Uncir nuestra expresión artística a la angustia local no es un modo de ablación. no será cortar las alas a nuestro escritor condenándolo a un localismo infecundo. a un arte alejado de toda superior entonación universalista. ${ }^{39}$ disposición por ampliar los paradigmas culturales de la realidad latinoamericana, que por un lado constituye una función dominante de su narrativa, y debe al discurso martiano un momento definitorio de enunciación de multintegralidad expresiva. Vale entonces reconocer en Carpentier, una legitimación de este proceso, a la vez que una actitud precursora en la cultura latinoamericana, por anticipar en este caso, la fase explicativa de un fenómeno culturologico que justo la contemporaneidad trata de reinterpretar. $Y$ así expresa:

Vi la posibilidad de establecer ciertos sincronismos posibles, americanos, recurrentes por encima del tiempo, relacionado esto con aquello, el ayer con el presente. Vi la posibilidad de traer ciertas verdades europeas a las latitudes que son nuestras actuando a contrapelo de quienes, viajando contra la trayectoria del ave. quisieron llevar verdades nuestras a donde, hace todavía treinta años, no había capacidad de entendimiento ni de medida para verlos en su justa dimensión. (Paulines Bonaparte fue, para mi lazarillo y guía, tiento primero -a partir de la Venus de Cánova- de los ensayos de indagación de los personajes que, como Billaud-Varenne, Collot d'Herbois, Victor Hugues, habrían de animar mi Siglo de las luces, visto en función de luces americanas. $)^{\text {4O }}$

De manera, que esta regularidad teórica dentro del discurso del ensayista cubano. lo sitúa en la avanzada de una dominante

\footnotetext{
${ }^{38}$ Ibidem, p. 84.

39 Marinello, Juan. Ensayos. La Habana. Editorial Arte y Literatura. 1977, p. 57.

${ }^{40}$ Op.cit, p. 76.
} 
cultural, donde es posible hallar una normativa a partir fundamentalmente de los procesos de intertextualidad y mitologización entre los más importantes de su creación literaria. Por otro lado. acerca de la utilidad y destreza técnica que le ofrece el dominio de diferentes tipos de arte, ya el escritor se había pronunciado por lo útil de contar con un "violín de Ingres" en el proceso creador.

Por lo tanto, en el volumen del discurso de Alejo Carpentier se estructura un cuerpo de ideas acerca de la actividad artística propiamente dicha, que además de constituir un elemento metodológico integrativo de su poética, deviene junto a la polifuncionalidad de su experiencia profesional, una clave determinante de importancia didáctica para el sujeto profesional del arte en la cultura artística latinoamericana, por la misma razón que significa el conocimiento esencial de su realidad, a través de una via potencial de interpretación e integración de más amplios paradigmas culturales.

Entonces, el diseño para una aproximación a un cuerpo teórico en relación con el discurso carpenteriano se estructura teniendo en cuenta un primer estudio acerca de los órdenes artísticas que se propone presentar, en suma, la pertinencia del pensamiento carpentiano en relación con la cultura -artística "...cuyo funcionamiento estético comunicativo tiene como consecuencia crear, conservar. difundir y asimilar los valores artísticos"41_ tareas éstas que en sí mismas corresponden al nivel operacional y conceptual de su prosa reflexiva, y donde nos proponemos explicitar sus razonamientos acerca del saber creador.
El segundo aspecto debe tratar sobre $\mathrm{El}$ Proceso Creador con el objetivo de desarrollar en el mismo un análisis descriptivo de las vertientes extra-literarias donde procede el pensamiento ideoestético carpenteriano, y sobre todo, en la importancia que tiene para el mismo un determinado sistema de principios en relación con la práctica del arte y en la que asume un papel de importancia el reconocimiento de la conciencia creadora.

En esta reelaboración de concientizar procesos y prácticas culturales, donde Carpentier propone un perenne ejercicio de recombinación que explícita como prioridad única, la necesaria comprensión por parte del sujeto artista de la contingencia que significa la simultaneidad del dominio de su modo de acción, por un lado, y la de superación de su realidad periferica por la otra. En las posibilidades de sistematizar todo el bagaje del pensamiento culturológico latinoamericano, de seguro podremos proyectar para el futuro, que sigue siendo el de nuestra incumplida modernidad, un acercamiento más exacto a la dimensión de nuestras más urgentes tareas de modelación y formación artísticas, y asimilar al mismo tiempo, las nuevas condiciones de prácticas y comunicación culturales -de las cuales ya hemos sido avisados por la revelación de nuestros más altos pensadores-, con la certeza de contar con una coherente y propia concentración de reflexión sobre nuestras realidades y pretensiones de creación, que bien supo advertir la fragua de esta acción intelectual excepcional de Alejo Carpentier, con el único interés de que el hombre - léase el artista- americano. pueda "mejorar lo que es".

\footnotetext{
41 Savranski, l: La cultura y sus funciones. Moscú. Editorial Progreso. 1983, p. 130.
} 